\title{
EXISTENTIALLY CLOSED LOCALLY COFINITE GROUPS
}

\author{
by FELIX LEINEN
}

(Received 23rd March 1990)

\begin{abstract}
Let $\mathfrak{X}$ be a class of finite groups. Then a $c \mathfrak{X}$-group shall be a topological group which has a fundamental system of open neighbourhoods of the identity consisting of normal subgroups with $\mathfrak{X}$-factor groups and trivial intersection. In this note we study groups which are existentially closed (e.c.) with respect to the class $L c \mathfrak{X}$ of all direct limits of $c \mathfrak{X}$-groups (where $\mathfrak{X}$ satisfies certain closure properties). We show that the so-called locally closed normal subgroups of an e.c. $L \boldsymbol{x}$-group are totally ordered via inclusion. Moreover it turns out

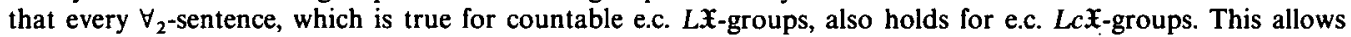
it to transfer many known properties from e.c. $L \mathfrak{X}$-groups to e.c. $L c \mathfrak{X}$-groups.
\end{abstract}

1980 Mathematics subject classification (1985 Revision): 20E26, 20E18, $22 \mathrm{A05}$.

\section{Introduction}

In this paper, $\mathfrak{X}$ will always denote a class of finite groups, which is closed with respect to subgroups, homomorphic images, and extensions. In particular, we write $\mathfrak{F}$, $\mathfrak{F}_{\pi}, \mathfrak{F}_{\pi} \cap \mathbb{G}, \mathfrak{F}_{p}$ for the classes of all finite groups, finite $\pi$-groups, finite soluble $\pi$-groups, and finite p-groups (resp.); here $\pi$ is a fixed set of primes. A great amount of information has been obtained about existentially closed (e.c.) groups in the class $L \mathfrak{X}$ of all locally $\mathfrak{X}$ groups, especially in the cases when $\mathfrak{X}=\mathfrak{F}$ or $\mathfrak{X}=\mathfrak{F}_{p}$ (see [7], [14, $\left.\S 6\right],[21],[15],[17]$, [18]). It was the original purpose of the present note to use this knowledge in studying e.c. locally residually $\mathfrak{X}$-groups ( $L R \mathfrak{X}$-groups). Here we cannot expect results as nice as in the $L \mathfrak{X}$-case. The reason is that there exist $2^{\aleph_{0}}$ finitely generated (f.g.) $R \mathfrak{F}_{p}$-groups ([5], [6]), hence also $2^{\aleph_{0}}$ countable e.c. $L R \mathfrak{X}$-groups (see also Example 4.5), while we have unique countable e.c. objects in $L F$ and in $L \mathfrak{F}_{p}$. However, the close connection between the classes $L \mathfrak{X}$ and $L R \mathfrak{X}$ is demonstrated by the fact, that there exist countable e.c. $L \mathfrak{X}$-groups, which are e.c. in $L R \mathfrak{X}$ (use the argument of [9, Satz 3.5]). If $\mathfrak{X}=\mathfrak{F}$ or $\mathfrak{X}=\mathfrak{F}_{p}$, then every e.c. $L \mathfrak{X}$-group is e.c. in $L R \mathfrak{X}$ (copy the proofs of [21, Satz 6] and [17, Theorem 3.7]).

Unfortunately, the study of e.c. $L R \mathfrak{X}$-groups is considerably complicated by the fact, that it seems to be very hard to find general constructions in order to produce sufficiently interesting $L R \mathfrak{X}$-supergroups of given $L R \mathfrak{X}$-groups. Here we are only able to use some ad hoc arguments. For example, $H N N$-extensions and free products of $L R F-$ groups stay in $L R F$, whenever the involved isomorphic subgroups are finite (see [1], [2]). The information obtained in this way is fairly weak.

Because of this situation, we put more emphasis on the profinite topologies living on each $R \mathfrak{X}$-group. This leads to the following refinement or our considerations. We replace 
$R \mathfrak{X}$ by the class $c \mathfrak{X}$ of all co-X-groups in the sense of [8]. These arise as follows. Let $U \in R \mathfrak{~}$. Suppose that $\mathscr{R}$ is a residual system in $U$, i.e., a set of normal subgroups in $U$ satisfying

(1) $U / N \in \mathfrak{X}$ for all $N \in \mathscr{R}$,

(2) for any $N_{1}, N_{2} \in \mathscr{R}$ there exists $N_{3} \in \mathscr{R}$ such that $N_{3} \leqq N_{1} \cap N_{2}$, and

(3) $\bigcap \mathscr{R}=1$.

Then $U$ becomes a Hausdorff topological space by requiring that $\mathscr{R}$ be a basis of neighbourhoods of the identity (the reader is referred to [11] or [10, §2] for the standard facts about topological groups). The topological group $U$ is now called a $c \mathfrak{X}$ group. Thus, a $c \mathfrak{X}$-group is just an $R \mathfrak{X}$-group equipped with a certain fixed pro- $\mathfrak{X}$ topology. In general, different residual systems on the same $R \mathfrak{X}$-group lead to different c: -groups.

If $U \leqq V \in c \mathfrak{X}$, then $U$ is a $c \mathfrak{X}$-group via the topology induced by $V$. For this reason, an embedding $\phi: U \rightarrow V$ of $c \mathfrak{X}$-groups shall be a group homeomorphism $\phi$ of $U$ onto the $c \mathfrak{x}$ subgroup $U \phi$ of $V$. Note that embeddings are always continuous. In order to prove that a monomorphism $\phi: U \rightarrow V$ of $c \mathfrak{X}$-groups is an embedding, it suffices to find residual systems $\mathscr{R}_{U}$ and $\mathscr{R}_{V}$ which give the topologies on $U$ resp. $V$ such that, for every $N \in \mathscr{R}_{U}$ there exists some $M \in \mathscr{R}_{V}$ with $M \cap U \phi \leqq N \phi$, and such that for every $M \in \mathscr{R}_{V}$ there exists some $N \in \mathscr{R}_{U}$ with $N \phi \leqq M \cap U \phi$. Since $\mathfrak{X}$-groups can only carry the discrete pro- $\mathfrak{X}$ topology, every monomorphism $\phi: U \rightarrow V$, where $U \in \mathfrak{X}$ and $V \in c \mathfrak{X}$, is an embedding. An $L c \mathfrak{X}$-group $G$ is a direct limit of $c \mathfrak{X}$-groups with respect to embeddings. Note that $G$ is in general not a topological group with respect to the direct limit topology, since multiplication in $G$ need not be continuous [3, Appendix 2, I.9]. However, with each f.g. subgroup of $G$ there is associated a unique topology. An embedding $\phi: G \rightarrow H$ of $L c \mathfrak{X}$-groups shall be a group monomorphism $\phi$ such that, for each f.g. subgroup $U$ of $G$, the restriction $\phi \mid U$ is a homeomorphism of $U$ onto $U \phi$. Again, monomorphisms $\phi: G \rightarrow H$, where $G \in L \mathfrak{X}$ and $H \in L c \mathfrak{X}$, are embeddings.

By the standard argument [13, Proposition 1.3], every $L c \mathfrak{X}$-group $G$ is embeddable into an e.c. $L c \mathfrak{X}$-group of cardinality $\max \left\{\boldsymbol{\aleph}_{0},|G|\right\}$. As before, there exist countable e.c. $L \mathfrak{X}$-groups which are e.c. in $L c \mathfrak{X}$. If $\mathfrak{X}=\mathfrak{F}$ or $\mathfrak{X}=\mathfrak{F}_{p}$, then every e.c. $L \mathfrak{X}$-group is e.c. in LcX.

Clearly, the class $p \mathfrak{X}$ of all pro- $\mathfrak{X}$-groups is a subclass of $c \mathfrak{X}$. Note that continuous monomorphisms of $p \mathfrak{X}$-groups are already embeddings [11, Proposition 10(F)]. On the other hand, every $c \mathfrak{X}$-group $U$ is a subgroup of its pro- $\mathfrak{X}$ completion $\hat{U}$ [8, Lemmata 2.5/6]. Let $\mathscr{L}$ be the local system of all f.g. subgroups in the $L \mathcal{A X}$-group $G$. If $X, Y \in \mathscr{L}$ satisfy $X \leqq Y$, then there exists an embedding $\alpha$ of $\hat{X}$ into $\hat{Y}$ such that the diagram

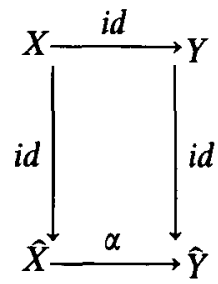


commutes [8, Theorem 2.1]. It follows, that $G$ is embeddable into its $L p \mathfrak{X}$-completion $\hat{G}=\lim \{\hat{X} \mid X \in \mathscr{L}\}$. Consequently, every e.c. $L p \mathfrak{X}$-group is e.c. in $L c \mathfrak{X}$, and the study of

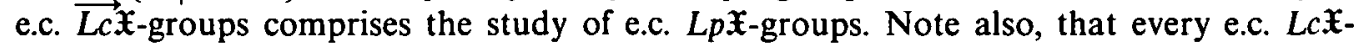
group is e.c. in its $L p \mathfrak{X}$-completion.

Our basic amalgamation technique within $L c \mathfrak{X}$ uses suitable factor groups of free products with amalgamation. A combination with embeddings of $c \mathfrak{X}$-groups into cartesian products of $\mathfrak{X}$-groups then reduces the problem of solving finite systems of equations and inequalities over an e.c. $L c \mathfrak{X}$-group $G$ to solving them over certain $\mathfrak{X}$ sections of f.g. subgroups of $G$. As a valuable corollary we note, that every $\forall_{2}$-sentence, which holds in every countable e.c. $L \mathfrak{X}$-group, is also true in every e.c. $L c \mathfrak{X}$-group.

From this, we can immediately carry over a lot of information from e.c. $L \mathfrak{X}$-groups to e.c. $L c \mathfrak{X}$-groups. For example, it follows that every e.c. $L c \mathfrak{F}$-group $G$ is simple. And isomorphisms between finite subgroups of $G$ are always induced by conjugation in $G$, while there can exist an element $g \in G$ of infinite order such that $g$ is conjugate to $g^{n}$ $(n \in \mathbb{Z})$ if and only if $n= \pm 1$ (see Section 6).

In the general case, we can carry over elementary properties like verbal completeness and triviality of centralizers of non-trivial normal subgroups. Also, unions and intersections of the so-called locally closed normal subgroups (see Section 3) of an e.c. $L c \mathfrak{X}$-group $G$ are totally ordered via inclusion. However, it remains open whether every normal subgroup of $G$ is such a union or intersection. In Section 4 we construct examples which show, that the $L \mathfrak{F}$-residual of $G$ can be a proper subgroup. The $L \&$ radical and the factor group modulo the $L F$-residual can be treated by methods used for e.c. LX-groups (see Section 3).

The theory becomes much more satisfactory in the case when $\mathfrak{X}=\mathfrak{F}_{p}$ (see Section 5). Here we can use our corollary about $\forall_{2}$-sentences directly to show, that every e.c. $L c F_{p}$-group has a unique chief series $\Sigma$, that the factors of $\Sigma$ are central and cyclic of order $p$, and that the order type of $\Sigma$ is a dense order without endpoints. If $K \supseteqq G$ satisfies $K \neq\left\langle g^{G}\right\rangle$ for all $g \in G$, then $K$ is e.c. in $G$. However, it seems to be unlikely that such a $K$ is in general e.c. in $L c \mathfrak{F}_{p}$ (as one might expect from the theory of e.c. $L \mho_{p}$-groups). We will show, that every subnormal subgroup of $G$ is already normal in $G$. Also, the results about embeddings of $L \mathfrak{F}_{p}$-groups into e.c. $L \mathfrak{F}_{p}$-groups $[15, \S 3]$ and about conjugacy of finite subgroups of e.c. $L \mathbb{F}_{p}$-groups [17, Theorem 6.1$]$ remain true for e.c. $L c F_{p}$-groups. As in the $L c F$-case, conjugacy of infinite f.g. subgroups of $G$ is more delicate. Although we can give a quite satisfactory necessary and sufficient condition (which shows for example, that an element $g \in G$ of infinite order is conjugate to $g^{n}(n \in \mathbb{Z})$ if and only if $\left.n \equiv 1(\bmod p)\right)$, it remains open whether an isomorphism $\phi: A \rightarrow B$ between f.g. subgroups of $G$ is induced by conjugation in $G$ if and only if $a^{-1} \cdot a \phi \in N$ for all $a \in(A \cap M)-N$ and all chief factors $M / N$ of $G$.

\section{Constructions within Lc:}

In the sequel, if $U \in c \mathfrak{X}$, then $N \unlhd_{0} U$ will denote that $N$ is an open normal subgroup of $U$. The following amalgamation theorem is the foundation of this note. 


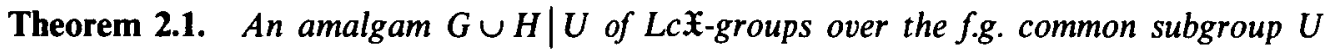
is embeddable into an LcX-group if and only if there exist local systems $\mathscr{L}_{G}$ in $G$ and $\mathscr{L}_{H}$ in $H$ consisting of f.g. subgroups which contain $U$ such that, for every pair $(X, Y) \in \mathscr{L}_{G} \times$ $\mathscr{L}_{H}$, the topologies on $X$ and $Y$ are given by residual systems $\mathscr{R}_{X, Y}$ in $X$ resp. $\mathscr{R}_{Y, X}$ in $Y$ such that, for every $L \in \mathscr{R}_{X, Y}$ (resp. $L \in \mathscr{R}_{Y, X}$ ), there exist $M \in \mathscr{R}_{X, Y}$ and $N \in \mathscr{R}_{Y, X}$ satisfying

(1) $M \leqq L$ (resp. $N \leqq L)$ and $M \cap U=L \cap U=N \cap U$, and

(2) the amalgam $X / M \cup Y / N \mid U L / L$ (where $U M / M$ and $U N / N$ are identified with $U L / L$ via $u M \equiv u L \equiv u N$ for all $u \in U$ ) is contained in an $\mathfrak{X}$-group.

Proof. Suppose first that the amalgam is contained in an $L c \mathfrak{X}$-group $W$. Let $\mathscr{L}_{W}, \mathscr{L}_{G}$ and $\mathscr{L}_{H}$ be the local systems of all f.g. subgroups in $W, G$ resp. $H$ containing $U$. For every $V \in \mathscr{L}_{W}$, denote by $\mathscr{R}_{V}$ the residual system of all $L \geqq_{0} V$. If $(X, Y) \in \mathscr{L}_{G} \times \mathscr{L}_{H}$, then let $\mathscr{R}_{X, Y}=\mathscr{R}_{\langle X, Y\rangle} \cap X$ and $\mathscr{R}_{Y, X}=\mathscr{R}_{\langle X, Y\rangle} \cap Y$. Since $X$ and $Y$ are $c \mathfrak{X}$-subgroups of $\langle X, Y\rangle$, the topologies on $X$ and $Y$ are given by the residual systems $\mathscr{R}_{X, Y}$ resp. $\mathscr{R}_{Y, X}$. Now every $M \in \mathscr{R}_{X, Y}$ (resp. $N \in \mathscr{R}_{Y, X}$ ) is induced by some $K \in \mathscr{R}_{\langle X, Y\rangle}$. Put $N=K \cap Y \in \mathscr{R}_{Y, X}$ (resp. $M=K \cap X \in \mathscr{R}_{X, Y}$ ). Then $M \cap U=N \cap U$, and the amalgam $X / M \cup Y / N \mid U M / M \equiv U N / N$ is embedded canonically in the $\mathfrak{X}$-group $\langle X, Y\rangle / K$.

Conversely, fix some $(X, Y) \in \mathscr{L}_{G} \times \mathscr{L}_{H}$ and regard the free product with amalgamation $F_{X, Y}=X_{U}^{*} Y$. Let

$$
\mathscr{R}_{X, Y}^{*}=\left\{K \supseteqq F_{X, Y} \mid F_{X, Y} / K \in \mathfrak{X}, \quad K \cap X \varrho_{0} X, \quad K \cap Y \supseteqq_{0} Y\right\}
$$

Denote epimorphic images modulo $\bigcap \mathscr{R}_{X, Y}^{*}$ by bars. Then $\bar{F}_{X, Y} \in c \mathfrak{X}$ with the topology given by the residual system $\overline{\mathscr{R}}_{X, Y}^{*}$. We will show:

An embedding of the amalgam $X \cup Y \mid U$ into $\bar{F}_{X, Y}$ is given by $z \rightarrow \bar{z}$ for all $z \in X \cup Y$.

If $X \leqq V \in \mathscr{L}_{G}$ and $Y \leqq W \in \mathscr{L}_{H}$, then id: $F_{X, Y} \rightarrow F_{V, W}$ induces an embedding of $\bar{F}_{X, Y}$ into $\bar{F}_{\boldsymbol{V}, \boldsymbol{W}}$.

Once (2.1) and (2.2) are proved, the discovered embeddings form commuting diagrams

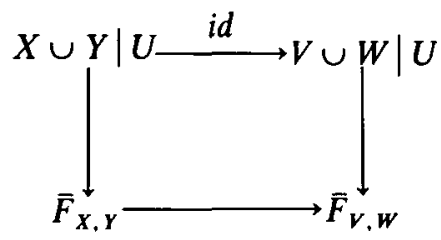


and therefore, the amalgam $G \cup H \mid U$ is embeddable into the direct limit of the $c \mathfrak{X}$ groups $\bar{F}_{X, Y}$ with respect to the embeddings given in (2.2).

To see (2.1), suppose that $1 \neq x \in X$. Then $x \notin L$ for some $L \in \mathscr{R}_{X, Y}$. By hypothesis, there exist $M \in \mathscr{R}_{X, Y}$ and $N \in \mathscr{R}_{Y, X}$ with $M \leqq L$ and $M \cap U=L \cap U=N \cap U$, and such that the amalgam $X / M \cup Y / N \mid U L / L$ is contained in an $\mathfrak{X}$-group $P$. Let $K$ be the kernel of the canonical homomorphism $F_{X, Y} \rightarrow P$. Clearly, $K \supseteqq F_{X, Y}$ with $\mathfrak{X}$-factor group such that $K \cap X=M \unlhd_{0} X$ and $K \cap Y=N \geqq_{0} Y$. Now $K \in \mathscr{R}_{X, Y}^{*}$ and $x \notin K$. It follows that $X \cap\left[\bigcap \mathscr{R}_{X, Y}^{*}\right]=1$, and so the canonical map $\phi: X \rightarrow \bar{X}$ is a group isomorphism. By definition of $\mathscr{R}_{X, Y}^{*}$, the map $\phi$ is continuous. The above argument also shows that we can find for every $L \in \mathscr{R}_{X, Y}$ some $K \in \mathscr{R}_{X, Y}^{*}$ with $K \cap X \leqq L$, whence $\phi$ is open.

In order to prove (2.2), it suffices to show:

$$
\check{K} \cap F_{X, Y} \in \mathscr{R}_{X, Y}^{*} \quad \text { for every } \check{K} \in \mathscr{R}_{\boldsymbol{V}, \boldsymbol{W}}^{*} \text {. }
$$

For every $K \in \mathscr{R}_{X, Y}^{*}$ there exists $\check{K} \in \mathscr{R}_{V}^{*}, W$ such that $\check{K} \cap F_{X, Y} \leqq K$.

The assertion (2.3) is an immediate consequence of the definition of $\mathscr{R}_{X, Y}^{*}$ and $\mathscr{R}_{V, W}^{*}$, and of the fact that the topology on $X$ resp. $Y$ is induced by the topology on $V$ resp. $W$. It remains to prove (2.4).

Let $K \in \mathscr{R}_{X, Y}^{*}$. Since $\mathscr{R}_{V, \mathrm{~W}} \cap X$ and $\mathscr{R}_{W, V} \cap Y$ are residual systems in $X$ resp. $Y$ which induce the topology on $X$ resp. $Y$, there exist $L_{1} \in \mathscr{R}_{V, W}$ and $L_{2} \in \mathscr{R}_{W, V}$ such that $L_{1} \cap X \leqq K \cap X$ and $L_{2} \cap Y \leqq K \cap Y$. From hypothesis, we obtain $M_{i} \in \mathscr{R}_{V, w}$ and $N_{i} \in \mathscr{R}_{W, V}$ such that $M_{1} \leqq L_{1}, N_{2} \leqq L_{2}$, and $M_{i} \cap U=L_{i} \cap U=N_{i} \cap U$, and such that the amalgam $V / M_{i} \cup W / N_{i} \mid U L_{i} / L_{i}$ is contained in an $\mathfrak{X}$-group $P_{i}$. Plainly, $M \cap U=N \cap U$ for $M=M_{1} \cap M_{2}$ and $N=N_{1} \cap N_{2}$. Denote epimorphic images modulo $M$ resp. $N$ by $\div$ Then an embedding $\alpha$ of the amalgam $\tilde{V} \cup \tilde{W} \mid \tilde{U}$ into $P_{3}=P_{1} \times P_{2} \in \mathfrak{X}$ is given by $\tilde{v} \alpha=\left(v M_{1}, v M_{2}\right)$ for all $v \in V$ and $\tilde{w} \alpha=\left(w N_{1}, w N_{2}\right)$ for all $w \in W$. Put $Q=F_{X, Y} / K$, and regard $\tilde{X} \cup \tilde{Y} \mid \tilde{U}$ as an amalgam in $P_{4}=Q \times P_{3}$ via $\tilde{z} \equiv(z K, \tilde{z} \alpha)$ for all $z \in X \cup Y$. We will now embed the amalgam $\tilde{V} \cup P_{4} \cup \tilde{W}$, where $\tilde{V} \cap P_{4}=\tilde{X}$ and $P_{4} \cap \tilde{W}=\tilde{Y}$, into an $\mathfrak{X}$-group.

To this end, let $P_{5}=Q \mathrm{Wr} P_{3} \in \mathfrak{X}$, and denote by $\pi_{1}: P_{4} \rightarrow P_{3}$ and $\pi_{2}: P_{5} \rightarrow P_{3}$ the canonical projections. Because of $\tilde{X} \cap Q=1$, we obtain from [12, Lemma 1] an embedding $\sigma: P_{4} \rightarrow P_{5}$ satisfying $\sigma \pi_{2}=\pi_{1}$ and $\left.\sigma\right|_{\hat{X}}=\left.\pi_{1}\right|_{\bar{X}}=\left.\alpha\right|_{\bar{X}}$. Lift $\sigma$ to an embedding of the amalgam $\tilde{V} \cup P_{4} \mid \tilde{X}$ into $P_{5}$ via $\left.\sigma\right|_{\mathscr{V}}=\left.\alpha\right|_{\tilde{F}}$. Regard the amalgam $P_{5} \cup \tilde{W} \sigma \mid \tilde{Y} \sigma$, where $\tilde{W} \sigma$ is an artificial copy of $\tilde{W}$. Denote the base group of $P_{5}$ by $\Omega$, and put $P_{6}=\Omega \mathrm{Wr} P_{3} \in \mathfrak{X}$. Since $\widehat{Y} \sigma \cap \Omega=1$, a further application of [12, Lemma 1] leads to an embedding $\tau: P_{5} \rightarrow P_{6}$ such that $\left.\sigma \tau\right|_{P}=\left.\sigma \pi_{2}\right|_{P}=\left.\pi_{1}\right|_{P}=\left.\alpha\right|_{p}$. Extend $\tau$ to an embedding of the amalgam $P_{5} \cup \tilde{W} \sigma \mid \tilde{Y} \sigma$ into $P_{6}$ via $\left.\sigma \tau\right|_{\tilde{W}}=\left.\alpha\right|_{W}$. Now $\sigma \tau$ embeds the amalgam $\tilde{V} \cup P_{4} \cup \tilde{W}$ into the $\mathfrak{X}$-group $P_{6}$.

Let $\breve{K}$ be the kernel of the group homomorphism $F_{V, W} \rightarrow P_{6}$ induced from $\tilde{\sigma} \sigma \tau$. Then $\check{K} \cap V=M$ and $\check{K} \cap W=N$, whence $\check{K} \in \mathscr{R}_{V}^{*}, w$. Moreover, by choice of $P_{4}$, we have $\check{K} \cap F_{X, Y} \leqq K$. 
Remark 2.2. Under the assumptions of Theorem 2.1, the amalgam $G \cup H \mid U$ is actually contained in an LcX-group $W$ with the following property: For all f.g. $X \leqq G$ and $Y \leqq H$ containing $U$, if $M \geqq_{0} X$ and $N \geqq_{0} Y$ are such that $M \cap U=N \cap U$ and such that the amalgam $X / M \cup Y / N \mid U M / M \equiv U N / N$ is contained in an $\mathfrak{X}$-group, then there exists $K \geqq_{0}\langle X, Y\rangle \leqq W$ such that $K \cap X=M$ and $K \cap Y=N$.

Proof. Since the amalgam is contained in an $L c \mathfrak{X}$-group, we may assume that $\mathscr{L}_{G}$ and $\mathscr{L}_{H}$ in Theorem 2.1 are the local systems of all f.g. subgroups in $G$ resp. $H$ which contain $U$. For $(X, Y) \in \mathscr{L}_{G} \times \mathscr{L}_{H}$, define $F_{X, Y}$ and $\mathscr{R}_{X, Y}^{*}$ as in the proof of Theorem 2.1. If $M \geqq_{0} X$ and $N \supseteqq_{0} Y$ satisfy the above assumptions, then the argument in the proof of (2.1) shows that there exists $K \in \mathscr{R}_{X, Y}^{*}$ with $K \cap X=M$ and $K \cap Y=N$.

Amalgams of $\mathfrak{F}$-groups are always contained in an $\mathfrak{F}$-group [22, Theorem 5.2]. Thus, if $\mathfrak{X}=\mathfrak{F}$, then the condition (2) in Theorem 2.1 becomes redundant. An amalgam $A \cup B \mid U$ of $\mathfrak{F}_{p}$-groups is contained in an $\mathfrak{F}_{p}$-group, if and only if there exist chief series in $A$ and $B$ which both induce the same chief series in $U$ (see [12]). In particular, amalgams of $\mathfrak{F}_{p}$-groups over a common cyclic subgroup are always contained in an $\mathfrak{F}_{p}$-group. Moreover, every cyclic group $U$ carries a unique pro- $\mathfrak{F}_{p}$ topology, and if $U \leqq V \in c \mathfrak{F}_{p}$, then the residual system of all $M \unlhd_{0} V$ induces the residual system of all $N \supseteqq_{0} U$ in $U$. Therefore, Theorem 2.1 yields that every amalgam of $L c \mathfrak{F}_{p}$-groups over a common cyclic subgroup is embeddable into an $L c F_{p}$-group.

In the case when $\mathfrak{X}=\mathfrak{F}_{p}$, it is readily verified that the conditions in Theorem 2.1 are equivalent to the property that, for each pair $(X, Y) \in \mathscr{L}_{G} \times \mathscr{L}_{H}$, the topologies on $X$ and $Y$ are given by descending chains $\mathscr{M}=\left\{M_{\alpha} \mid \alpha \in \omega\right\}$ and $\mathscr{N}=\left\{N_{\alpha} \mid \alpha \in \omega\right\}$ of open normal subgroups $M_{\alpha} \supseteqq X, N_{\alpha} \supseteqq Y$ of index $\leqq p^{\alpha}$ such that $\bigcap \mathscr{M}=1=\bigcap \mathscr{N}$ and $\mathscr{M} \cap U=$ $\mathscr{N} \cap U$. Therefore our criteria for amalgamation within $L c \mathfrak{F}$ resp. $L c \mathfrak{F}_{p}$ are in line with the criteria given in [23, Theorems 1.2 and 3.1] for the existence of the pro- $₹$ resp. pro- $\mathfrak{F}_{p}$ amalgamated product. In fact, it follows from [8, Theorem 2.1], that the pro- $\mathfrak{X}$ completion $E_{X, Y}$ of the group $\bar{F}_{X, Y}$ in the proof of Theorem 2.1 is the pro- $\mathfrak{X}$ amalgamated product of $\hat{X} \cup \hat{Y} \mid \hat{U}$. In particular, the conditions in Theorem 2.1 ensure the existence of the pro-X amalgamated product of $\hat{X} \cup \hat{Y} \mid \hat{U}$. Moreover, $(2.1) /(2.2)$ and $[8$, Theorem 2.1] lead to commuting diagrams

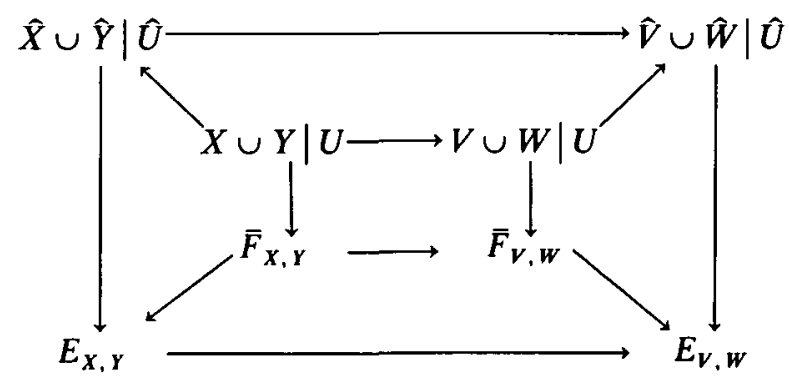

of the canonical embeddings, and so $\hat{G} \cup \hat{H} \mid \hat{U}$ is embedded canonically in $\underset{\longrightarrow}{\lim } E_{X, \gamma}$. 
Theorem 2.1 allows us to deduce a necessary and sufficient criterion for the solvability of finite systems of equations and inequalities in e.c. $L c \mathfrak{X}$-groups.

Theorem 2.3. A finite system of equations and inequalities with coefficients $c_{1}, \ldots, c_{r}$ in the e.c. LcX-group $G$ has a solution in $G$ if and only if there exists a local system $\mathscr{L}$ in $G$ consisting of f.g. subgroups which contain $U=\left\langle c_{1}, \ldots, c_{r}\right\rangle$ such that, for every $X \in \mathscr{L}$, the topology on $X$ is given by a residual system $\mathscr{R}_{X}$ such that, for every $M \in \mathscr{R}_{X}$, the system $\mathscr{S} / M$ (with coefficients $c_{1} M, \ldots, c_{r} M$ ) has a solution in some $\mathfrak{X}$-group $W_{X, M} \geqq X / M$.

Proof. Suppose that $g_{1}, \ldots, g_{s}$ is a solution to $\mathscr{S}$ in $G$. Let $\mathscr{L}$ be the local system of all f.g. subgroups of $G$ which contain $V=\left\langle U, g_{1}, \ldots, g_{s}\right\rangle$. For $X \in \mathscr{L}$, denote by $\mathscr{R}_{X}$ the residual system of all $M \unlhd_{0} X$ such that $w\left(c_{1}, \ldots, c_{r}, g_{1}, \ldots, g_{s}\right) \notin M$ for every inequality $w\left(c_{1}, \ldots, c_{r}, x_{1}, \ldots, x_{s}\right) \neq 1$ in $\mathscr{S}$. Clearly, if $M \in \mathscr{R}_{X}$. then $\mathscr{S} / M$ has the solution $g_{1} M, \ldots, g_{s} M$ in $W_{X, M}=X / M$.

Conversely, let $\mathscr{M}=\left\{(X, M) \mid M \in \mathscr{R}_{X}, X \in \mathscr{L}\right\}$. Regard $H=\prod\left\{W_{X, M} \mid(X, M) \in \mathscr{M}\right\}$ as a $c \mathfrak{X}$-group under the product topology (where each $W_{X, M}$ carries the discrete topology). Denote by $K_{X, M}$ the obvious direct complement to $W_{X, M}$ in $H$. An embedding $\phi: U \rightarrow H$ is given by

$$
u \phi=(u M)_{(X, M) \in \mathcal{M}} \text { for all } u \in U
$$

since $K_{X, M} \cap U \phi=(M \cap U) \phi$ for all $(X, M) \in \mathscr{M}$. In the following, we suppress $\phi$ and regard $U$ as a subgroup of $H$. Because $H$ contains the componentwise solution to $\mathscr{S}$, and because $G$ is e.c. in $L c \mathfrak{X}$, it suffices to embed the amalgam $G \cup H \mid U$ into an $L c \mathfrak{X}$ group. To this end, we will check the conditions of Theorem 2.1.

Put $\mathscr{L}_{G}=\mathscr{L}$, and let $\mathscr{L}_{H}$ be the local system in $H$ of all f.g. subgroups containing $U$. For $(X, Y) \in \mathscr{L}_{G} \times \mathscr{L}_{H}$, choose $\mathscr{R}_{X, Y}=\mathscr{R}_{X}$ and $\mathscr{R}_{Y, X}=\left\{N \geqq_{0} Y \mid N \cap U \in \mathscr{R}_{X} \cap U\right\}$. If $L \supseteqq_{0} Y$, then there exists $M \in \mathscr{R}_{X}$ such that $M \cap U \leqq L \cap U$, whence $N=L \cap K_{X, M} \in \mathscr{R}_{Y, X}$ satisfies $N \leqq L$. This shows that $\mathscr{R}_{Y, X}$ is a residual system in $Y$ which gives the topology on $Y$.

If $M \in \mathscr{R}_{X, Y}$, then $N=K_{X, M} \cap Y \in \mathscr{R}_{Y, X}$ satisfies $M \cap U=N \cap U$, and the amalgam $X / M \cup Y / N \mid U M / M \equiv U N / N$ can be embedded into $W_{X, M}$ via $x M \rightarrow x M$ for all $x \in X$ and $y K_{X, M} \rightarrow y_{X, M}$ for all $y \in Y$ (where $y_{X, M}$ denotes the component of $y \in \boldsymbol{H}$ in $\boldsymbol{W}_{X, M}$ ). Finally, regard some $L \in \mathscr{R}_{Y, X}$. Then $M \cap U=L \cap U$ for some $M \in \mathscr{R}_{X, Y}$, and $N=$ $K_{X, M} \cap L \in \mathscr{R}_{Y, X}$ satisfies $N \leqq L$ and $L \cap U=N \cap U$. Denote epimorphic images modulo $M$ resp. $N$ by bars. It remains to embed the amalgam $\bar{X} \cup \bar{Y} \mid \bar{U}$ into an $\mathfrak{X}$-group. Identify $\bar{Y}$ with a subgroup of $P_{1}=W_{X, M} \times Y / L \in \mathfrak{X}$ via $\bar{y} \equiv\left(y_{X, M}, y L\right)$ for all $y \in Y$. Since $\bar{U} \cap Y / L=1$, we obtain from [12, Lemma 1] an embedding $\sigma: P_{1} \rightarrow P_{2}=Y / L$ Wr $W_{X, M} \in \mathfrak{X}$ satisfying $\bar{u} \sigma=\bar{u} \in W_{X, M}$ for all $u \in U$. Extend $\sigma$ to an embedding of the amalgam $\bar{X} \cup P_{1} \mid \bar{U}$ into $P_{2}$ via $\bar{x} \sigma=\bar{x} \in W_{X, M}$ for all $x \in X$. Then $P_{2}$ embeds in particular the amalgam $\bar{X} \cup \bar{Y} \mid \bar{U}$. 
Lemma 2.4. Suppose that $\mathscr{R}=\mathscr{R}_{1} \cup \mathscr{R}_{2}$ is a residual system in the $R \mathfrak{X}$-group $U$. Then $\mathscr{R}_{1}$ or $\mathscr{R}_{2}$ is a residual system in $U$ which gives the same topology on $U$ as $\mathscr{R}$.

Proof. It suffices to show that one of the sets $\mathscr{R}_{1}$ and $\mathscr{R}_{2}$ contains for every $N \in \mathscr{R}$ some $M$ such that $M \leqq N$. Assume that there exists $N \in \mathscr{R}_{1}$ such that no $M \in \mathscr{R}_{2}$ is contained in $N$. Let $\widetilde{\mathscr{R}}=\{M \in \mathscr{R} \mid M \leqq N\}$. Clearly $\widetilde{\mathscr{R}} \subseteq \mathscr{R}_{1}$, and thus $\mathscr{R}_{1}$ has the desired property.

Using the method of construction given in [9, Satz 3.5], every $\mathfrak{X}$-group can be embedded into a countable e.c. $L \mathfrak{X}$-group, which is e.c. in $L c \mathfrak{X}$. It is therefore possible to formulate the following corollary.

Corollary 2.5. The $\forall_{2}$-sentences, which hold in every e.c. LcX-group, are precisely the

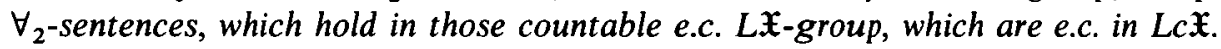

Note that the classes $L \mathbb{F}$ and $L \mathbb{F}_{p}$ contain a unique countable e.c. group.

Proof. Let $\psi$ be an $\forall_{2}$-sentence, i.e., let $\psi=\forall \bar{x}\left(\phi_{1}(\bar{x}) \vee \cdots \vee \phi_{n}(\bar{x})\right)$ where $\phi_{i}(\bar{x})$ is a primitive formula for every $i$. Suppose that one of the countable e.c. $L \mathfrak{X}$-group $E$, which are e.c. in $L c \mathfrak{X}$, satisfies $\psi$. Regard any e.c. $L c \mathfrak{X}$-group $G$ and some $\bar{c}$ from $G$. Denote by $\mathscr{L}$ the local system of all f.g. subgroups of $G$ containing $\bar{c}$.

Fix some $X \in \mathscr{L}$. Let $\mathscr{R}_{i}$ be the set of all $M \varrho_{0} X$ such that $\phi_{i}(\overline{c M})$ holds in some X-group containing $X / M$. If $M \supseteqq_{0} X$, then $X / M \times E \in L c X$, and so the group $E$ contains a copy of $X / M$. Since $E \models \psi$, it follows that $M$ lies in some $\mathscr{R}_{i}$. Hence Lemma 2.4 yields that one of the $\mathscr{R}_{i}$ is a residual system in $X$ which induces the topology on $X$.

This shows that $\mathscr{L}=\mathscr{L}_{1} \cup \cdots \cup \mathscr{L}_{n}$ where $\mathscr{L}_{i}$ is the set of all $X \in \mathscr{L}$ in which the topology is given by a residual system $\mathscr{R}_{X}$ such that, for every $M \in \mathscr{R}_{X}$, there exists an $\mathfrak{X}$-group $W \geqq X / M$ with $W \models \phi_{i}(\overline{\mathrm{cM}})$. By $[14$, Lemma $1 . \mathrm{A} .10]$, one of the $\mathscr{L}_{i}$ is a local system in $G$. Now Theorem 2.3 applied to this local system yields that $G \models \phi_{i}(\vec{c})$. This shows that $G \models \psi$.

As a second embedding technique within $L c \mathfrak{X}$, we will adopt the construction of $[18$, $\S 2]$ as far as possible.

Construction 2.6. Let ${ }^{-}: G \rightarrow H$ be a homomorphism with kernel $N$. For every f.g. $X \leqq G$, let $W_{X}=X \mathrm{Wr} H$ (unrestricted wreath product). Fix some f.g. $U \leqq G$, and choose left transversals $R$ of $U \cap N$ in $U$, and $T$ of $\bar{U}$ in $H$. Then a group monomorphism

$$
\tau: G \longrightarrow W=\bigcup\left\{W_{X} \mid X \leqq G \text { f.g. }\right\}
$$

is given by $g \tau=f_{g} \cdot \bar{g}$, where the function $f_{g}: H \rightarrow\langle g, U\rangle$ is defined via $\left(t_{1} \bar{r}_{1}\right) f_{g}=r_{1} g r_{2}^{-1}$ whenever $t_{i} \in T, r_{i} \in R$ satisfy $t_{2} \bar{r}_{2}=t_{1} \bar{r}_{1} \bar{g}$.

Theorem 2.7. Adopt the notation of Construction 2.6. Let $\mathscr{L}_{G}$ and $\mathscr{L}_{H}$ be the local systems of all f.g. subgroups in $G$ resp. $H$. Suppose that $G \in L c \mathfrak{X}, H \in L \mathfrak{X}$, and that 
$N \cap X \unlhd_{0} X$ for all $X \in \mathscr{L}_{G}$. Regard the base group $\Omega_{X}$ of $W_{X}$ as a cX-group under the product topology. The groups $W_{X, Y}=\Omega_{X} \times Y$, where $(X, Y) \in \mathscr{L}_{G} \times \mathscr{L}_{H}$, form a local system in $W$. Each $W_{X, Y}$ is a c: $\mathfrak{X}$-group under the topology given by the residual system

$$
\mathscr{R}_{X, Y}=\left\{\operatorname{core}_{W, Y}(K) \mid K \geqq_{0} \Omega_{X}\right\}=\left\{L \supseteqq{ }_{0} \Omega_{X} \mid L \supseteqq W_{X, Y}\right\}
$$

With these topologies, $\tau: G \rightarrow W$ becomes an embedding of Lcx-groups.

Proof. If $\left(X_{i}, Y_{i}\right) \in \mathscr{L}_{G} \times \mathscr{L}_{H}$ with $X_{1} \leqq X_{2}$ and $Y_{1} \leqq Y_{2}$, then the topology on $X_{2}$ induces the topology on $X_{1}$, and so the product topology on $\Omega_{X_{2}}$ induces the product topology on $\Omega_{X_{1}}$. Therefore, id: $W_{X_{1}, Y_{1}} \rightarrow W_{X_{2}, Y_{2}}$ is an embedding. This shows that $W$ is an $L c \mathfrak{X}$-group.

Now, fix some $X \in \mathscr{L}_{G}$ with $U \leqq X$. It remains to show that $\left.\tau\right|_{X}: X \rightarrow X \tau \leqq W_{X, \bar{X}}$ is a homeomorphism. To this end, we consider the residual system $\mathscr{R}_{X}=\left\{M \varrho_{0} X \mid M \leqq\right.$ $N \cap X\}$ in $X$. If $x \in N \cap X$, then $x \tau=f_{x}$ where $(t \vec{r}) f_{x}=r x r^{-1}$ for all $t \in T, r \in R$. Therefore,

$$
L_{M}=\{f: H \rightarrow X \mid(\bar{X}) f \subseteq M\} \in \mathscr{R}_{X, \bar{X}}
$$

satisfies $L_{M} \cap X \tau=M \tau$ for every $M \in \mathscr{R}_{X}$, and thus $\left.\tau\right|_{X}$ is open. To see that $\left.\tau\right|_{X}$ is continuous, let $L \in \mathscr{R}_{X, \bar{X}}$. Then there exist $h_{1}, \ldots, h_{v} \in H$ and $M_{1}, \ldots, M_{v} \geqq_{0} X$ such that

$$
L \geqq\left\{f: H \rightarrow X \mid\left(h_{i}\right) f \in M_{i} \text { for } \quad 1 \leqq i \leqq v\right\} .
$$

Put $M=\bigcap\left\{M_{i} \mid 1 \leqq i \leqq v\right\} \varliminf_{0} X$. Then $M \tau \leqq L \cap X \tau$.

\section{General results}

Let us begin with some elementary applications of Corollary 2.5 . We will denote by $\pi$ the set of all primes which divide the order of some $\mathfrak{X}$-group.

Theorem 3.1. The following assertions hold for every e.c. LcX-group G.

(a) $G$ is verbally complete.

(b) If $1 \neq N \leqq G$, then $\mathbb{C}_{G}(N)=1$.

(c) If $U$ and $V$ are f.g. subgroups of $G$, then $\left[U, V^{g}\right]=1$ for some $g \in G$.

(d) Every non-trivial normal subgroup of $G$ contains for every $\pi$-number $\mu$ an element of order $\mu$.

(e) For each $\pi$-number $\mu$, every element of $G$ is in the normal closure of some element of order $\mu$.

Proof. Let $E$ be any countable e.c. $L \mathfrak{X}$-group. Then parts $(\mathrm{a})-(\mathrm{c})$ hold for $E$ by $[16$, Theorem 2.1 and p. 212], and by the argument of [15, Lemma 2.2]. Note that (b) can be encoded by the $\forall{ }_{2}$-sentence $\forall g, h \exists x\left(g \neq 1 \neq h \rightarrow\left[g, h^{x}\right] \neq 1\right)$. For the proof of (d) and (e), regard the $\forall_{2}$-sentences 


$$
\forall g \exists x, y\left[\bigwedge_{k=1}^{\mu-1} x^{k} \neq 1 \wedge x^{\mu}=1 \wedge(g \neq 1 \rightarrow x=[g, y])\right]
$$

and

$$
\forall g \exists x, y, z\left[\bigwedge_{k=1}^{\mu-1} x^{k} \neq 1 \wedge x^{\mu}=1 \wedge g=[x, y, z]\right]
$$

These hold for $E$ by the arguments of [16, Lemmata 2.5/4.3(b) and 3.3]. Therefore, Corollary 2.5 applies in all cases.

Because every f.g. $c\left(\mathfrak{F}_{\pi} \cap(\mathfrak{F})\right.$-group $H$ is hypoabelian, it follows from the argument of [14, Proposition 1.B.3] that minimal normal subgroups of $L c\left(\mathfrak{F}_{\pi} \cap(\mathfrak{G})\right.$-groups are abelian. Hence Theorem 3.1(b) implies that e.c. $L c\left(\mathscr{F}_{\pi} \cap(5)\right.$-groups have no minimal normal subgroups.

Next, let us extend the notion of a $G$-subgroup given in [21, p. 114] for $L \mathfrak{F}_{p}$-groups. Suppose that $\mathscr{L}$ is the local system of all f.g. subgroups of an $L c \mathfrak{X}$-group $G$. Then we say that $N$ is a $G$-subgroup of $U \in \mathscr{L}\left(N \leqq{ }_{G} U\right)$, if every $V \in \mathscr{L}$ with $U \leqq V$ contains some $M \geqq_{0} V$ such that $M \cap U=N$. Put $U_{G}^{*}=\bigcap\left\{N \mid N \geqq_{G} U\right\} \supseteqq U$. It is readily verified that $U \leqq V \in \mathscr{L}$ and $N \geqq_{G} V$ implies $N \cap U \unlhd_{G} U$, and so we may form

$$
G^{*}=\bigcup\left\{U_{G}^{*} \mid U \in \mathscr{L}\right\} \supseteqq G .
$$

Note that $G^{*} \leqq \bigcap\left\{N \supseteqq G \mid N \cap U \supseteqq{ }_{0} U\right.$ for all $\left.U \in \mathscr{L}\right\}$. A subgroup $\tilde{N}=\bigcup\left\{N_{X} \mid X \in \mathscr{L}\right\}$ is said to be a locally closed normal subgroup in $G$, if every $N_{X}$ is a closed normal subgroup in $X$, and if $N_{X} \leqq N_{Y}$ whenever $X \leqq Y$. Every $N \leqq G$ is contained in the locally closed normal subgroup $\tilde{N}=\bigcup\left\{N_{X} \mid X \in \mathscr{L}\right\}$, where $N_{X}=\bigcap\left\{K \geqq_{0} X \mid N \cap X \leqq K\right\}$ is the closure of $N \cap X$ in $X$. Another kind of locally closed normal subgroup $\tilde{N}$ can be obtained from each $N \geqq_{G} U \in \mathscr{L}$ by choosing $N_{X}=\bigcap\left\{K \geqq_{0} X \mid K \cap U=X \cap N\right\}$. In the latter case $\tilde{N} \cap U=N$, and we immediately have:

Lemma 3.2. Let $N \geqq_{G} U$ where $U$ is a f.g. subgroup of the LcX-group $G$. Then $\left\langle N^{G}\right\rangle \cap U=N$.

Theorem 3.3. Let $\tilde{N}=\bigcup\left\{N_{X} \mid X \in \mathscr{L}\right\}$ be a locally closed normal subgroup of the e.c. LcX-group $G$. Then the following hold for every $g \in G-\tilde{N}$.

(a) Every finite system of equations and inequalities with coefficients from $\tilde{N}$, which is solvable in $G$, already has a solution in every verbal subgroup of $\left\langle g^{G}\right\rangle$. In particular, $\tilde{N} \leqq\left\langle g^{G}\right\rangle$, the locally closed normal subgroups of $G$ are totally ordered via inclusion, and the $G$-subgroups in each f.g. subgroup of $G$ form a descending chain of length $\leqq x$.

(b) If $0(\tilde{N} g)=n<\infty$, then any two elements in $\tilde{N} g$ of order $n$ are conjugate in every verbal subgroup of $\left\langle g^{G}\right\rangle$.

Proof. (a) Let $\mathscr{S}$ be a finite system of equations and inequalities with coefficients 
$c_{1}, \ldots, c_{r} \in \tilde{N}$, unknowns $x_{1}, \ldots, x_{s}$, and a solution $g_{1}, \ldots, g_{s} \in G$. Fix any reduced word $v\left(\xi_{1}, \ldots, \xi_{t}\right) \neq 1$. Adjoin to $\mathscr{S}$ the equations

$$
x_{i}=v\left(\xi_{i 1}, \ldots, \xi_{i t}\right) \quad \text { and } \quad \xi_{i j}=\prod_{k=1}^{3}\left[g, y_{i j k}, z_{i j k}\right] \quad(1 \leqq i \leqq s, 1 \leqq j \leqq t)
$$

with coefficients $g$. In the case when $0(\tilde{N} g)=n<\infty$, let $h_{1}, h_{2} \in \tilde{N} g$ with $n=0\left(h_{i}\right)$, and adjoin the additional equations

$$
\begin{gathered}
h_{1}^{x_{s+1}}=h_{2}, \quad x_{s+1}=v\left(\xi_{s+1,1}, \ldots, \xi_{s+1, t}\right), \quad \text { and } \\
\xi_{s+1, j}=\prod_{k=1}^{3}\left[g, y_{s+1, j, k}, z_{s+1, j, k}\right](1 \leqq j \leqq t)
\end{gathered}
$$

with coefficients $g, h_{1}, h_{2}$. Denote the resulting system by $\mathscr{T}$.

Since $\tilde{N}$ is locally closed, there exists a f.g. $V \leqq G$ with $g_{1}, \ldots, g_{s}, g \in V$ and $c_{1}, \ldots, c_{r} \in N v$ (and with $h_{1}, h_{2} \in N_{V} g$ in the case when $\left.n<\infty\right)$. Let $\mathscr{L}_{G}=\{X \in \mathscr{L} \mid V \leqq X\}$. Because $N_{X}$ is closed in $X \in \mathscr{L}_{G}$, there exists $L_{X} \unlhd_{0} X$ with $N_{X} \leqq L_{X}$ and $g \notin L_{X}$ (and with $\left\langle h_{i}\right\rangle \cap L_{X}=1$ in the case when $\left.n<\infty\right)$. Regard in $X$ the residual system $\mathscr{R}_{X}$ of all $M \geqq_{0} X$ satisfying $M \leqq L_{X}$ and $w\left(c_{1}, \ldots, c_{r}, g_{1}, \ldots, g_{s}\right) \notin M$ for every inequality $w\left(c_{1}, \ldots, c_{r}, x_{1}, \ldots, x_{s}\right) \neq 1$ in $\mathscr{S}$. Fix some $M \in \mathscr{R}_{X}$. Since every e.c. LX-group is verbally complete, we can find an $\mathfrak{X}$-group $F_{X, M} \geqq X / M$ such that, for every $x \in X$, there exist elements $f_{x, l} \in F_{X, M}$ with $x M=v\left(\left[f_{x, 1}, f_{x, 2}\right], \ldots,\left[f_{x, 2 t-1}, f_{x, 2 t}\right]\right)$. Identify $X / M$ with its image in $L_{X} / M$ Wr $X / L_{X} \leqq W_{X, M}=F_{X, M} \mathrm{Wr} X / L_{X}$ under some Krasner-Kaloujnine embedding.

Let $m_{X}=0\left(L_{X} g\right)$, and choose a left transversal $T$ of $\left\langle L_{X} g\right\rangle$ in $X / L_{X}$. Then every $f: X / L_{X} \rightarrow F_{X, M}$ can be decomposed in $W_{X, M}$ into a product $f=f_{1} f_{2} f_{3}$, where

$$
\begin{aligned}
& \operatorname{supp} f_{1} \subseteq T \\
& \operatorname{supp} f_{2} \subseteq \bigcup\left\{T \cdot\left(L_{X} g^{i}\right) \mid i \in\left\{1, \ldots, m_{X}-1\right\} \text { is odd }\right\} \\
& \operatorname{supp} f_{3} \subseteq \bigcup\left\{T \cdot\left(L_{X} g^{i}\right) \mid i \in\left\{1, \ldots, m_{X}-1\right\} \text { is even }\right\} .
\end{aligned}
$$

With this decomposition, the arguments of [16, Theorem 4.7 and Lemmata 4.2/4.3] actually show that the system $\mathscr{T} / M$ with coefficients $M c_{1}, \ldots, M c_{r}, M g$ (and $M h_{1}, M h_{2}$ ) has a solution in $W_{X, M}$. Therefore, Theorem 2.3 yields that $\mathscr{T}$ has a solution in $G$, whence $\mathscr{S}$ (and the equation $h_{1}^{x}=h_{2}$ ) have a solution in the verbal subgroup of $\left\langle g^{G}\right\rangle$ generated by $v\left(\xi_{1}, \ldots, \xi_{t}\right)$.

Finally, if $h \in \tilde{N}$, then an application of the above to the system $\mathscr{S}$ consisting only of the equation $h=x$ with coefficient $h$ shows that $h \in\left\langle g^{G}\right\rangle$. Hence $\bar{N} \leqq\left\langle g^{G}\right\rangle$.

Note, that Theorem 3.3 does in fact hold for unions and intersections of locally closed normal subgroups. 
Question. Is every normal subgroup of an e.c. Lcæ-group the union or intersection of a chain of locally closed normal subgroups?

If $N \supseteqq G \in L c \mathfrak{X}$ is such that $N \cap U$ is closed in $U$ for every f.g. $U \leqq G$, then each $U / U \cap N$ is a $c \mathbb{X}$-group via the quotient topology. Note however, that this is in general not enough to ensure that $G / N \in L c \mathfrak{X}$ since, for $U \leqq V$, the canonical monomorphism $U /(U \cap N) \rightarrow V /(V \cap N)$ need not be an embedding [11, p. 23].

By combining Theorem 2.1 with [15, Theorem 2.1], we can apply the technique of $[16, \S 2]$ in order to extend [16, Theorems 2.3-2.6] to the normal $L \&$-subgroups of an e.c. $L c \mathfrak{X}$-group $G$. In particular, the normal $L \xi$-subgroups of $G$ form a chain. We will now turn to the $L \&$-quotients of $G$. Note that by Theorem 3.3, for every f.g. subgroup $U$ of an e.c. $L c$ X-group $G$, we have $G^{*} \cap U=U_{G}^{*}$ or $G^{*} \cap U \supseteqq_{G} U$.

Theorem 3.4. Let $G$ be e.c. in Lcx.

(a) If $N \leqq G$, then $N \leqq G^{*}$ or $G^{*} \leqq N$.

(b) If $G^{*}<N \leqq G$, then $N \cap U \leqq{ }_{G} U$ for every f.g. $U \leqq G$. In particular, $G / N \in L X$.

(c) If $G / G^{*}$ has a minimal normal subgroup, then (b) also applies to $N=G^{*}$.

(d) $G^{*}=\bigcap\left\{N \supseteqq G \mid N \cap U \geqq_{0} U\right.$ for all f.g. $\left.U \leqq G\right\}$. In particular, $G^{*}$ contains the LX-residual of $G$.

(e) If for every infinite f.g. $U \leqq G$ the chain of $G$-subgroups in $U$ has length $\omega$, then $G^{*}$ is the LF-residual of $G$.

Proof. (a) Suppose that $N$ is not in $G^{*}$. Fix some $g \in N-G^{*}$. Since $G^{*}$ is a locally closed normal subgroup in $G$, Theorem 3.3 yields $G^{*} \leqq\left\langle g^{G}\right\rangle \leqq N$.

(b) Since $G^{*}<N$, we may assume that $G^{*} \cap U<N \cap U$. Choose $M \varrho_{G} U$ maximal with respect to $N \cap U \nsubseteq M$. Choose $g \in(N \cap U)-M$. Then Theorem 3.3 yields $M \leqq$ $\left\langle g^{G}\right\rangle \cap U \leqq N \cap U$, whence $N \cap U \geqq_{0} U$. Since this also holds for every f.g. $V \leqq G$ containing $U$, we even obtain $N \cap U \varrho_{G} U$.

(c) Let $N / G^{*}$ be a minimal normal subgroup in $G / G^{*}$. Fix some $g \in N-G^{*}$. Regard any f.g. $U \leqq G$ containing $g$. From (b) we have $N \cap U \geqq_{G} U$. Choose $M \unlhd_{G} U$ maximal with respect to $G^{*} \cap U \leqq M<N \cap U$. Now $\left\langle M^{G}\right\rangle \cap U=M$ by Lemma 3.2. Because $N / G^{*}$ is minimal normal in $G / G^{*}$, we conclude from (a) that $\left\langle M^{G}\right\rangle \leqq G^{*}$. But then $G^{*} \cap U=M \supseteqq_{G} U$.

(d) We have already noted that $G^{*} \leqq S=\bigcap\left\{N \leqq G \mid N \cap U \leqq_{\mathbf{0}} U\right.$ for all f.g. $\left.U \leqq G\right\}$. Assume that $G^{*}<S$. Then (b) implies that $S / G^{*}$ is a minimal normal subgroup in $G / G^{*}$. But now (c) leads to $S \leqq G^{*}$, a contradiction.

(e) Let $N \leqq G$ with $G / N \in L F$. Then, for every infinite f.g. $U \leqq G$, we have $|U: U \cap N|<\infty$. By our assumption, $U \cap N$ is therefore not contained in the intersection of all $G$-subgroups of $U$. Choose $M \triangleq_{G} U$ maximal with respect to $U \cap N \nsubseteq M$. As in (b), $U \cap N \geqq_{0} U$. The latter also holds for finite $U$. It thus follows from (d) that $G^{*} \leqq N$.

Theorem 3.4 enables us to apply Construction 2.6 to all normal subgroups of an e.c. 
$L c \mathfrak{X}$-group $G$ which contain $G^{*}$ (see Theorem 2.7). We can therefore extend most of the results from [15] and [18] about normal subgroups and chief factors of e.c. $L \mathfrak{X}$-groups to normal subgroups and chief factors of $G$ above $G^{*}$. Here is a list of theorems which can be transposed literally: [15, Theorems 2.3, 2.5 and 2.6], [18, Theorems 3.1-4.3]. Also, $[15, \S 3]$ and the corresponding results about embeddings of countable supersoluble $\pi$-groups into e.c. $L\left(\mathscr{F}_{\pi} \cap(\mathfrak{G})\right.$-groups carry over (cf. [18, end]). As a slight extension of [15, Theorem 2.5] we note that, if $N \leqq G$ contains an element $h$ of infinite order, then so does each coset of $N$ in $G$ (apply Theorem 3.1(c) with $U=\langle x\rangle$ and $V=\langle h\rangle$; then $h^{g} x$ is the desired element in the coset $N x$ ). Finally, [16, Theorem 5.1] about locally inner automorphisms of countable e.c. $L \mathfrak{X}$-groups holds as well for countable e.c. $L c \mathfrak{X}$-groups.

\section{4. $G$-subgroups}

Up to now, we have not established the existence of proper $G$-subgroups in infinite f.g. subgroups of an $L c \mathfrak{X}$-group $G$. In fact, they need not exist, since we will show in Theorem 6.1 that e.c. $L c \mathfrak{F}$-groups are simple. However, the situation is not always so bad.

Theorem 4.1 Suppose that all simple $\mathfrak{X}$-groups are contained in a variety $\mathscr{V}$ such that every free $\mathscr{V}$-group of finite rank has only finitely many subgroups of finite index. Then every infinite f.g. subgroup $U$ of an Lc: $\mathfrak{X}$-group $G$ contains a descending chain of $G$ subgroups of length $\omega$.

Proof. Suppose that $M \unlhd_{G} U$. Let $\mathcal{N}$ be the set of all $N \geqq_{0} M$ containing $\mathscr{V}(M)$. Then $\mathscr{N}$ is finite by hypothesis. Assume that $\mathscr{N}$ contains no $G$-subgroup of $U$. Then we can find a f.g. $X \leqq G$ such that $U \leqq X$, and such that $L \cap U \notin \mathscr{N}$ for all $L \leqq \varrho_{0} X$. On the other hand, there exists $K \geqq_{0} X$ with $K \cap U=M$, and we can choose $L \unlhd_{0} X$ maximal with respect to $L<K$. As a chief factor of $X / L \in \mathfrak{X}$, the factor $K / L$ lies in $\mathscr{V}$. But now $L \cap U \in \mathcal{N}$, a contradiction. This shows that the desired chain can be constructed inductively.

Theorem 4.1 applies for example in the case when $\mathfrak{X}=\mathfrak{F}_{\pi} \cap \mathfrak{G}$ for a finite set $\pi$ of primes. More generally, Theorem 4.1 holds whenever the exponents of the simple $\mathfrak{X}$ groups are uniformly bounded (use Zelmanov's solution of the restricted Burnside problem [24], [25]).

Because chief factors of $\xi_{p}$-groups are cyclic of order $p$, Theorem 4.1 has the following Corollary.

Corollary 4.2. Every f.g. subgroup $U$ of an $L \subset F_{p}$-group $G$ contains $G$-subgroups of every index $p^{\alpha} \leqq|U|(\alpha \in \omega)$.

This of course generalizes the fact, that every chief series of an $L F_{p}$-group induces a chief series on each of its finite subgroups. Conversely, we know from [15, Corollary 
3.3], that every series of an $\mathfrak{F}_{p}$-group $U$ is induced by the unique chief series of some countable e.c. $L \mathscr{F}_{p}$-group $G \geqq U$. We will also extend this result to the $L c \mathfrak{F}_{p}$-case.

Construction 4.3. Let $\left(n_{\alpha}\right)_{\alpha<\omega}$ be a sequence with $n_{\alpha} \leqq \omega$. Suppose that $U_{0}$ is an infinite f.g. $c \mathfrak{F}_{p}$-group, and that $\left\{U_{0 \beta} \mid \beta<\omega\right\}$ is a descending chain of open normal subgroups of index $p^{\beta}$ in $U_{0}$. Then there exists an ascending chain $\left\{U_{\alpha} \mid \alpha<\omega\right\}$ of f.g. ç्Fp-groups, and for every $\alpha$ a descending chain $\left\{U_{\alpha \beta} \mid \beta<\omega\right\}$ of open normal subgroups of index $p^{\beta}$ in $U_{\alpha}$ such that

(1) $G=\bigcup\left\{U_{\alpha} \mid \alpha<\omega\right\}$ is an e.c. LcF $\mathfrak{F}_{p}$-group,

(2) $\left\{U_{\alpha \beta} \mid \beta<\omega\right\}$ is the unique chain of $G$-subgroups in $U_{\alpha}$,

(3) for every $\beta \leqq n_{\alpha}$, there exists $\beta^{\prime}<\omega$ such that $U_{\alpha \beta}=U_{\alpha} \cap U_{\alpha+1, \beta^{\prime}}$, and

(4) if $n_{\alpha}<\omega$, and if $m_{\alpha}$ is minimal with respect to $U_{\alpha, n_{\alpha}}=U_{\alpha} \cap U_{\alpha+1, m_{\alpha}}$, then $U_{\alpha, n_{\alpha}}=$ $U_{\alpha} \cap U_{\alpha+1, \beta^{\prime}}$ for all $\beta^{\prime} \geqq m_{\alpha}$.

In the course of the construction it suffices to choose $n_{\alpha+1}$ after determining $U_{\alpha}$ and the chain $\left\{U_{\alpha \beta} \mid \beta<\omega\right\}$.

Proof. (a) Using Cantor's diagonal enumeration of $\omega \times \omega$ we can find a bijection $\chi: \omega \times \omega \rightarrow \omega$ such that $\alpha \leqq(\alpha, \beta) \chi$ for all $\alpha, \beta<\omega$ (see also [9, Satz 3.5]). The construction is now performed inductively in such a way that,

$$
\text { for all } \alpha^{\prime} \leqq \alpha, \beta<\omega \text {, there exists } N \geqq_{0} U_{\alpha} \text { such that } U_{\alpha^{\prime} \beta}=N \cap U_{\alpha^{\prime}} \text {. }
$$

In the step $\alpha \rightarrow \alpha+1$, let $\mathscr{S}_{\alpha \gamma}, \gamma<\omega$, be an enumeration of all finite systems of equations and inequalities with coefficients from $U_{\alpha}$. Fix $i, j<\omega$ with $\alpha=(i, j) \chi$. Then $\alpha \geqq i$, and $\mathscr{S}_{i j}$ does already exist by induction. If $\mathscr{S}_{i j}$ is solvable in a f.g. $c \mathscr{F}_{p}$-group $V \geqq U_{\alpha}$ such that,

$$
\text { for all } \alpha^{\prime} \leqq \alpha, \beta<\omega \text {, there exists } N \leqq_{0} V \text { such that } U_{\alpha^{\prime} \beta}=N \cap U_{\alpha^{\prime}},
$$

then put $V_{\alpha}=V$; otherwise, let $V_{\alpha}=U_{\alpha}$. It is now easy to find a descending chain $\left\{V_{\alpha \beta} \mid \beta<\omega\right\}$ of open normal subgroups of index $p^{\beta}$ in $V_{\alpha}$ such that $\left\{U_{\alpha \beta} \mid \beta \leqq n_{\alpha}\right\} \subseteq$ $\left\{U_{\alpha} \cap V_{\alpha \beta} \mid \beta<\omega\right\}$. If $n_{\alpha}=\omega$, then we put $U_{\alpha+1}=V_{\alpha}$ and $U_{\alpha+1, \beta}=V_{\alpha \beta}$ for all $\beta<\omega$. In this case, (4.1) and (3) are satisfied, while (4) is empty. Otherwise, we identify $V_{\alpha}$ with the first factor of $U_{\alpha+1}=V_{\alpha} \times V_{\alpha}$, regard $U_{a+1}$ as a $c F_{p}$-group via the product topology, and define

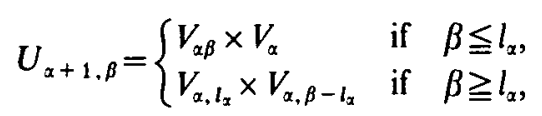

where $l_{\alpha}$ is minimal with respect to $U_{\alpha, n_{\alpha}}=U_{\alpha} \cap V_{\alpha, l_{z}}$. Then (3), (4) and (4.1) hold, and the induction is completed.

(b) Fix some $\alpha, \beta<\omega$ and $g \in U_{\alpha}-U_{\alpha \beta}, h \in U_{\alpha \beta}$. We will show now that the system $\mathscr{S}$ consisting only of the equation 


$$
h=\prod_{j=1}^{\nu}\left[g, x_{j}, y_{j}\right] \quad \text { where } \quad \nu=0\left(U_{\alpha \beta} g\right)
$$

has a solution in $G$. Clearly, $\mathscr{S}=\mathscr{S}_{\alpha \gamma}$ for some $\gamma<\omega$. Let $\mu=(\alpha, \gamma) \chi$. Because of the construction given in (a) it suffices to show that $\mathscr{S}$ has a solution in some f.g. $c F_{p}$-group $V \geqq U_{\mu}$ satisfying (4.2).

Because of (4.1) there exists $L \geqq_{0} U_{\mu}$ such that $L \cap U_{\alpha}=U_{\alpha \beta}$. Therefore, $\mathscr{R}_{\mu}=$ $\left\{M \varrho_{0} U_{\mu} \mid M \leqq L\right\}$ is a residual system in $U_{\mu}$ which gives the topology on $U_{\mu}$. Since every e.c. $L c \mathfrak{F}_{p}$-group is verbally complete, there exists a f.g. $c \mathfrak{F}_{p}$-group $P \geqq\langle h\rangle$ such that $h$ is a commutator in $P$. Applying Theorem 2.1 and Remark 2.2. to the amalgam $U_{\alpha \beta} \cup P \mid\langle h\rangle$, we obtain a f.g. $c F_{p}$-group $F \geqq U_{\alpha \beta}$ such that $h$ is a commutator in $F$, and such that

every $N \bigcap_{0} U_{\alpha \beta}$ is induced in $U_{\alpha \beta}$ by some open normal subgroup of $F$.

Let $\sigma: U_{\alpha} \rightarrow U_{\alpha \beta} \mathrm{Wr} U_{\alpha} / U_{\alpha \beta} \leqq W=F \mathrm{Wr} U_{\alpha} / U_{\alpha \beta}$ be a Krasner-Kaloujnine embedding. Denote the base group of $W$ by $\Omega$. For $K \Xi_{0} F$, let $\check{K}=\{f \in \Omega \mid \operatorname{Im} f \subseteq K\}$. Note that, for $M \in \mathscr{R}_{\mu}$, we have

$$
\check{K} \cap U_{\alpha} \sigma=\left(M \cap U_{\alpha}\right) \sigma \text { if and only if } K \cap U_{\alpha \beta}=M \cap U_{\alpha \beta}
$$

In particular, if $\mathscr{R}_{W}=\left\{\check{K} \mid K \geqq_{0} F\right.$ with $K \cap U_{\alpha \beta}=M \cap U_{\alpha \beta}$ for some $\left.M \in \mathscr{R}_{\mu}\right\}$, then $\mathscr{R}_{W} \cap U_{\alpha} \sigma=\left(\mathscr{R}_{\mu} \cap U_{\alpha}\right) \sigma$. Put $R=\bigcap \mathscr{R}_{W}$, and regard $H=W / R$ as a $c \mathscr{F}_{p}$-group under the topology given by the residual system $\mathscr{R}_{H}=\mathscr{R}_{W} / R$. Then the composition of $\sigma$ andthe canonical epimorphism $W \rightarrow H$ embeds $U_{\alpha}$ into the $c \mathfrak{F}_{p}$-group $H$. In the following, we identify $U_{\alpha}$ with its image in $H$ under this embedding. Now the proof of [16, Lemma 4.3(b)] actually shows that $\mathscr{S}$ has a solution in $H$. Our aim is to obtain the desired group $V$ from an application of Theorem 2.1 to the amalgam $U_{\mu} \cup H \mid U_{\alpha}$.

To this end, regard some $K \geqq_{0} F$ and $M \in \mathscr{R}_{\mu}$ with $K \cap U_{\alpha \beta}=M \cap U_{\alpha \beta}$. Because of (4.3) and (4.4), we can find for any chief series of open normal subgroups in $U_{\mu}$, which refines $1 \leqq M \leqq L<U_{\mu}$, a chief series of open normal subgroups in $H$, which refines $1 \leqq \breve{K} / R \leqq \Omega / R \leqq H$, such that both series induce the same on $U_{a}$. It thus follows from [12] that the amalgam $U_{\mu} / M \cup H / N \mid U_{\alpha} M / M \equiv U_{\alpha} N / N$, where $N=\check{K} / R$, is contained in an $\mathfrak{F}_{p}$-group. This shows that we may apply Theorem 2.1 and Remark 2.2 to find a f.g. $c F_{p}$-group $V$ containing the amalgam $U_{\mu} \cup H \mid U_{\alpha}$, and satisfying

whenever $M \bigoplus_{0} U_{\mu}$ and $N \geqq_{0} H$ are such that $M \cap U_{\alpha}=N \cap U_{\alpha}$ and such that amalgam $U_{\mu} / M \cup H / N \mid U_{a} M / M \equiv U_{a} N / N$ is contained in an $\mathfrak{F}_{p}$-group, then there exists $K \leqq_{0} V$ with $K \cap U_{\mu}=M$ and $K \cap H=N$.

It remains to prove that $V$ satisfies (4.2).

To this end, fix $\alpha \leqq \kappa \leqq a$. From (4.1) there exists a descending chain $\left\{M_{\lambda} \mid \lambda<\omega\right\}$ of open normal subgroups in $U_{\mu}$ which induces $\left\{U_{\kappa \lambda} \mid \lambda<\omega\right\}$ in $U_{\kappa}$, and hence also $\left\{U_{a \beta^{\prime}} \mid \beta^{\prime} \leqq n_{\alpha}\right\}$ in $U_{\alpha}$. Therefore, (4.3) and (4.4) yield a descending chain of open normal 
subgroups in $H$ which induces $\left\{U_{\alpha \beta^{\prime}} \mid \beta^{\prime} \leqq n_{\alpha}\right\}$ in $U_{a}$. Now (4.5) gives $K_{\lambda} \unlhd_{0} V$ with $K_{\lambda} \cap U_{\mu}=M_{\lambda}$. Suppose now that $\kappa<\alpha$. By the above argument, there exists a descending chain $\left\{K_{\lambda} \mid \lambda \leqq n_{\kappa}\right\}$ of open normal subgroups in $V$ which induces $\left\{U_{\kappa \lambda} \mid \lambda \leqq\right.$ $\left.n_{\kappa}\right\}$ in $U_{\kappa}$. If $\lambda>n_{\kappa}$, then (3) and (4) yield $U_{\kappa \lambda} \leqq U_{\alpha \beta}$, and using (4.1) we can extend $\left\{K_{\lambda} \cap U_{\mu} \mid \lambda \leqq n_{\kappa}\right\}$ to a descending chain $\left\{M_{\lambda} \mid \lambda<\omega\right\}$ of open normal subgroups in $U_{\mu}$, which induces $\left\{U_{\kappa \lambda} \mid \lambda<\omega\right\}$ in $U_{\kappa}$, and which satisfies $M_{\lambda} \in \mathscr{R}_{\mu}$ for all $\lambda>n_{\kappa}$. Again, (4.3)-(4.5) apply.

(c) Clearly, (4.1) ensures that every $U_{\alpha \beta}, \beta<\omega$, is a $G$-subgroup of $U_{\alpha}$. Conversely, if $N \unlhd_{G} U_{\alpha}$, then $N$ has finite index in $U_{\alpha}$. Therefore, $N$ is not contained in $\bigcap\left\{U_{\alpha \beta} \mid \beta<\omega\right\}$. Choose $\beta$ minimal with respect to $N \nsubseteq U_{a, \beta+1}$. If $g \in N-U_{a, \beta+1}$, then (b) and Lemma 3.2 yield that $U_{\alpha, \beta+1}\left\langle\left\langle g^{G}\right\rangle \cap U_{\alpha} \leqq\left\langle N^{G}\right\rangle \cap U_{\alpha}=N \leqq U_{\alpha \beta}\right.$. It follows that $N=U_{\alpha \beta}$. This shows that (2) holds.

(d) Finally, let us prove (1). Suppose, that $\mathscr{S}$ is a finite system of equations and inequalities with coefficients $g_{1}, \ldots, g_{r} \in G$ and a solution $h_{1}, \ldots, h_{s}$ in some $L c \mathfrak{F}_{p}$-supergroup $H$ of $G$. Choose $\alpha<\omega$ such that $g_{1}, \ldots, g_{r} \in U_{\alpha}$. Then $\mathscr{S}=\mathscr{S}_{\alpha \gamma}$ for some $\gamma<\omega$. Let $\mu=(\alpha, \gamma) \chi$, and regard $V=\left\langle U_{\mu}, h_{1}, \ldots, h_{s}\right\rangle$. By the construction given in (a), $\mathscr{S}$ will have a solution in $U_{\mu+1} \leqq G$ if, for all $\kappa \leqq \mu, \lambda<\omega$, there exists $N \leqq_{0} V$ such that $U_{\kappa \lambda}=N \cap U_{\kappa}$. But this is true, since the $U_{\kappa \lambda}$ are the only $G$ - and hence also the only $H$-subgroups of $U_{\kappa}$ (Corollary 4.2).

Corollary 4.4. Let $U$ be an infinite f.g. $c F_{p}$-group. If $\left\{N_{\alpha} \mid \alpha<\omega\right\}$ is a descending chain of open normal subgroups of index $p^{\alpha}$ in $U$, then $U$ is contained in a countable e.c. $L c \mathfrak{F}_{p}$-group $G$ such that the $N_{\alpha}$ are precisely the G-subgroups in $U$.

Construction 4.3 enables us to build various examples of countable e.c. $L c \mathfrak{F}_{p}$-groups.

Example 4.5. Each of the following properties is shared by $2^{\aleph_{0}}$ (pairwise non-

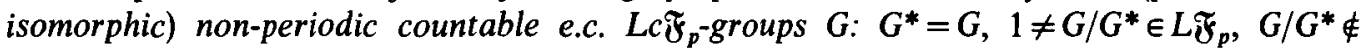
$L \mathfrak{Z}_{p}$.

Proof. Regard the free group $U_{0}$ of rank two as a $c \mathfrak{F}_{p}$-group under the topology given by the residual system of all $N \leqq U_{0}$ of $p$-power index. Since the commutator factor group of every subgroup of finite index in $U_{0}$ is free abelian of rank $\geqq 2[20$, Proposition I.3.9], there exist $2^{\aleph_{0}}$ descending chains of subgroups $\left\{U_{0 \beta} \mid \beta<\omega\right\}$ of index $p^{\beta}$ in $U_{0}$ with $\bigcap\left\{U_{0 \beta} \mid \beta<\omega\right\}=1$. An application of Construction 3.4 to these chains clearly yields $2^{\mathrm{N}_{0}}$ countable e.c. $L c \mathfrak{f}_{p}$-groups $G$, which are not isomorphic as $L c \mathfrak{F}_{p}$-groups, since they contain $U_{0}$ in too many non-compatible ways. Choose $n_{\alpha}=0$ (resp. $n_{\alpha}=\omega$ ) for all $\alpha$ to establish $G^{*}=G$ (resp. $G / G^{*} \notin L \mathfrak{F}_{p}$ ). Moreover, if $0<n_{0}<\omega$, then $n_{\alpha+1}=m_{\alpha}$ for all $\alpha$ leads to $1 \neq G / G^{*} \in L \mathfrak{\xi}_{p}$, since $U_{0, n_{0}-1} G^{*} / G^{*}$ is a minimal normal subgroup in $G / G^{*}$ in this case.

\section{E.c. $L c \mathfrak{F}_{p}$-groups}

In the case when $\mathfrak{X}=\mathfrak{F}_{p}$, we can make more progress by considering further 
applications of Corollary 2.5 to e.c. $L c \mathfrak{F}_{p}$-groups. To this end we will have to regard specific $\forall_{2}$-sentences. For any reduced word $w\left(\xi_{1}, \ldots, \xi_{v}\right) \neq 1$, we define the term $t_{w}(g, \bar{x})$ as follows.

$$
t_{w}(g, \bar{x})=w\left(\prod_{i=0}^{p-1}\left[g, x_{1, i}, x_{1, p+i}\right], \ldots, \prod_{i=0}^{p-1}\left[g, x_{v, i}, x_{v, p+i}\right]\right)
$$

Then the $\forall_{2}$-sentence $\phi_{w}$ is given by

$$
\phi_{w}=\forall g, h \exists \bar{x}\left[\bigvee_{k=0}^{p-1} h=g^{k} \cdot t_{w}(g, \bar{x}) \vee \bigvee_{k=0}^{p-1} g=h^{k} \cdot t_{w}(h, \bar{x})\right]
$$

Lemma 5.1. Every e.c. $L c \mathfrak{F}_{p}$-grouup satisfies the above $\forall_{2}$-sentences $\phi_{w}$.

Proof. Note that $\left[x_{1 j}, x_{2 j}\right]=\left[(b, f), x_{1 j}, x_{2 j}\right]$ actually holds for all $j \in\{1, \ldots, m\}$ in the proof of [16, Lemma 4.3(b)]. Therefore, a detailed analysis of the proof of [16, Theorem 4.7] shows that the unique countable e.c. $L \mathfrak{F}_{p}$-group satisfies each $\phi_{w}$. Now Corollary 2.5 applies.

Lemma 5.1 provides the key for the proof of the following theorem.

Theorem 5.2. Every e.c. $L c \mathfrak{F}_{p}$-group $G$ has a unique chief series $\Sigma$. The chief factors of $G$ are central and cyclic of order $p$, and the order type of $\Sigma$ is a dense order without endpoints.

Proof. Regard any $K, L \leqq G$. Suppose that there exists $g \in K-L$. From $g \notin L$ we have $g \notin\left\langle h^{G}\right\rangle$ for every $h \in L$. Therefore it follows from Lemma 5.1 that $h \in\left\langle g^{G}\right\rangle$ for every $h \in L$, whence $L \leqq K$. This shows that the normal subgroups of $G$ are totally ordered via inclusion. Equivalently, $G$ has a unique chief series.

Regard a chief factor $M / N$ in $G$. If $g \in M-N$ then, from Lemma 3.2, $\Sigma$ must induce the unique chain of $G$-subgroups in $\langle g\rangle$, when $g^{p} \in N$. Therefore, $\exp (M / N)=p$. Regard the reduced word $w(\xi)=\xi^{p}$ and some $g, h \in M-N$. Then Lemma 5.1 yields that $g \in h^{k} N$ or $h \in g^{k} N$ for some $k \in\{1, \ldots, p-1\}$. This shows that $M / N$ is cyclic of order $p$. Since $G$ is verbally complete, $G$ has no finite epimorphic image, and so $M / N$ must be central.

Lemma 5.1 applied to the word $w\left(\xi_{1}, \xi_{2}\right)=\left[\xi_{1}, \xi_{2}\right]$ yields that $M^{\prime}=N$ for every chief factor $M / N$ in $G$. Since $G$ is also perfect and has trivial centre (Theorem 3.1), we may deduce as in the proof of $[19$, Theorem $B(d)]$ that $\Sigma$ has the desired order type.

Theorem 5.2 implies that every $L c \mathfrak{F}_{p}$-group has a chief series with central and cyclic factors of order $p$. Note also, that the existence of a unique chief series with dense order type and elementary-abelian factors could also be shown for e.c. $L c\left(\mathfrak{F}_{\pi} \cap \mathfrak{G}\right)$-groups, where $\pi$ is a finite set of primes, if there would exist a pendant to Lemma 5.1, i.e., if it could be shown, for example, that there exists some fixed $m<\omega$ such that, for every 
chief factor $M / N$ of a countable e.c. $L\left(\mathfrak{F}_{\pi} \cap(\mathfrak{5})\right.$-group, and for all $g, h \in M-N$, the element $N h \in G / N$ is a product of at most $m$ conjugates of powers of $N g$ in $G / N$ (cf. [16, Theorem 4.9 and p. 214]).

Theorem 5.3. Let $K \leqq G$, where $G$ is e.c. in $L c \mathfrak{F}_{p}$, and suppose that $K \neq\left\langle g^{G}\right\rangle$ for all $g \in G$. Then the following hold.

(a) $K$ is e.c. in $G$. In particular, every normal subgroup of $K$ is already normal in $G$, and conjugation with elements from $G$ induces locally inner automorphisms on $K$.

(b) If $K \in L \mathfrak{F}_{p}$, then $K$ is e.c. in $L \mathfrak{F}_{p}$.

Proof. Let $\mathscr{S}$ be a finite system of equations and inequalities with coefficients $\bar{c}$ and unknowns $\bar{x}$.

(a) Denote by $\phi(\bar{c}, \bar{x})$ the conjunction of all equations and inequalities from $\mathscr{S}$. Consider the $\forall_{2}$-sentence

$$
\forall g, \bar{c}, \bar{d}, \bar{y}_{i} \exists h, \bar{z}_{j}\left[g \neq 1 \wedge \phi(\bar{c}, \bar{d}) \wedge \bigwedge_{i} c_{i}=t_{w}\left(g, \bar{y}_{i}\right) \rightarrow \phi(\bar{c}, \bar{h}) \wedge \bigwedge_{j} h_{j}=t_{w}\left(g, \bar{z}_{j}\right)\right]
$$

where $w\left(\xi_{1}, \xi_{2}\right)=\left[\xi_{1}, \xi_{2}\right]$. This sentence expresses that, whenever $\mathscr{S}$ has coefficients $\bar{c}$ in $[g, G, G]^{\prime}$ and a solution $\bar{d}$ in $G$, then there exists a solution $\bar{h}$ to $\mathscr{S}$ in $\left\langle g^{G}\right\rangle$. It is satisfied in the countable e.c. $L \oiint_{p}$-group [16, Theorem 4.8 ]. Thus Corollary 2.5 , Lemma 5.1 and Theorem 5.2 yield that the groups $N$ in the chief factors $M / N$ of $G$ are e.c. in $G$. It now follows from the arguments of [16, Theorem 4.8], that $K$ satisfies (a).

(b) Suppose that $\mathscr{P}$ has coefficients in $K$ and a solution in the $L \mathscr{F}_{p}$-group $H \geqq K$. Then $U=\langle\bar{c}\rangle$ is finite. Since $K$ is e.c. in $G$, and since $G$ is e.c. in $L c \mathscr{F}_{p}$, it suffices to embed the amalgam $G \cup H \mid U$ into an $L c \oiint_{p}$-group. To this end, we check the conditions of Theorem 2.1. By (a), $K$ has a unique chief series. So [21, Hilfssatz 1] yields the existence of a finite group $V \geqq U$ in $K$ such that every chief series in $V$ induces the $K$ chief series in $U$. Let $\mathscr{L}_{G}$ and $\mathscr{L}_{H}$ be the local systems in $G$ resp. $H$ of all f.g. subgroups containing $V$. For $(X, Y) \in \mathscr{L}_{G} \times \mathscr{L}_{H}$, put $\mathscr{R}_{X, Y}=\left\{M \leqq_{0} X \mid M \cap V=1\right\}$ and $\mathscr{R}_{Y, X}=\{1\}$. Then [12] ensures that, for all $M \in \mathscr{R}_{X, Y}$, the amalgam $X / M \cup Y \mid U M / M \equiv U$ is contained in an $\mathfrak{F}_{p}$-group, since $X / M \geqq V M / M \cong V$ and $Y \geqq V$ enforce that all chief series in $X / M$ and $Y$ induce the $K$-chief series in $U M / M \equiv U$.

It remains open, whether Theorem 5.3(b) can be extended to arbitrary $K$. Note that, if $K$ is the unique countable e.c. $L \oiint_{p}$-group $E_{p}$, then $G$ is contained canonically in the group LokInn $\left(E_{\mathrm{p}}\right)$ of all locally inner automorphisms of $E_{p}$ by Theorem 3.1(b).

Theorem 5.4. Let $G$ be e.c. in $L c \mathfrak{F}_{p}$. Then every subnormal subgroup of $G$ is already normal in $G$.

Proof. Assume that there exists a subnormal subgroup $S$ of defect 2 in $G$. Because of Theorem 5.3, there exists a chief factor $M / N$ in $G$ such that $S \triangleleft M \triangleleft G$, and such that $S$ is not in $N$. Regard the $\forall_{2}$-sentence 


$$
\forall g, h, \bar{x} \exists \bar{y}, \bar{z}_{i}\left[h=t_{w}(g, \bar{x}) \rightarrow h=t_{v}(g, \bar{y}) \wedge \bigwedge_{i} y_{i}=t_{w}\left(g, \bar{z}_{i}\right)\right]
$$

where $v(\xi)=\xi$ and $w\left(\xi_{1}, \xi_{2}\right)=\left[\xi_{1}, \xi_{2}\right]$. A detailed analysis of the proof of [16, Theorem $4.11(\mathrm{f})]$ shows that this sentence is satisfied by the countable e.c. $L \mathfrak{F}_{p}$-group. Hence it also holds in $G$ by Corollary 2.5. Because of Lemma 5.1 and Theorem 5.2 we conclude that $N \leqq[g, N, N] \leqq S<M$ for any $g \in S-N$. But this enforces $S=N$, a contradiction.

We can also extend [15, §3] and [16, Theorems 4.1/2] literally to results about embeddings of countable $L \mathfrak{F}_{p}$-groups into e.c. $L c F_{p}$-groups and to results about partial complements to normal subgroups $\neq\left\langle g^{G}\right\rangle$ in e.c. $L c \mathfrak{F}_{p}$-groups $G$ (here, $G_{0} / N$ resp. $G_{0} / K$ must be in $L \mathscr{F}_{p}$ ). To this end we just transform the systems of equations and inequalities used in the proofs of these theorems into suitable $\forall_{2}$-sentences. Example 4.5 shows that the full generalization of the above embedding results (without restriction to $L \mathfrak{F}_{p}$-groups) does not hold. As far as conjugacy of f.g. subgroups in e.c. $L c \mathfrak{F}_{p}$-groups is concerned, we have the following result.

Theorem 5.5. Let $G$ be an e.c. $L c \mathfrak{F}_{p}$-group.

(a) An isomorphism $\phi: A \rightarrow B$ between f.g. subgroups of $G$ is induced by conjugation in $G$, if and only if there exists a local system $\mathscr{L}$ in $G$ of f.g. subgroups which contain $\langle A, B\rangle$ such that, in every $X \in \mathscr{L}$, there is a chief series $\left\{N_{a}\right\}_{a \in \omega}$ of open normal subgroups such that $a^{-1} \cdot a \phi \in N_{\alpha+1}$ for all $a \in\left(A \cap N_{\alpha}\right)-N_{\alpha+1}$.

(b) An element $g \in G$ of infinite order is conjugate in $G$ to $g^{n}(n \in Z)$, if and only if $n \equiv 1$ $(\bmod p)$.

Proof. Combine Theorem 2.3 with [15, Corollary 3.3(b)] and [17, Theorem 6.1].

It remains open whether every automorphism of $G$, which stabilizes the unique chief series in $G$, is a locally inner automorphism (cf. [17, Theorem 6.1]).

\section{E.c. LcF-groups}

The techniques developed in Section 2 yield the following informations about e.c. LcF-groups.

Theorem 6.1. The following assertions hold for every e.c. LcF-group G.

(a) Every isomorphism between finite subgroups of $G$ is induced by conjugation in $G$.

(b) If $G \cup H \mid U$ is an amalgam of $G$ with a countable LF-group $H$ over a finite subgroup $U$, then id: $U \rightarrow G$ can be extended to an embedding $H \rightarrow G$.

(c) For all $g, h \in G-1$ there exist $x, y \in G$ such that $h=g^{x} g^{y}$. In particular, $G$ is simple.

(d) On every f.g. abelian subgroup of $G$, inversion is induced by conjugation in $G$. In particular, every element in $G$ is conjugate to its inverse. 
(e) Let $w_{1}, \ldots, w_{n}$ be words in unknowns $x_{1}, \ldots, x_{n}$ and elements of $G$. Denote by $d_{i j}$ the exponent sum of $x_{j}$ in $w_{i}$. If $\operatorname{det}\left(d_{i j}\right) \neq 0$, then the system of equations $w_{i}=1$ for $1 \leqq i \leqq n$ has a solution in $G$.

Proof. The assertions (a), (c), (d), (e) can be encoded as $\forall_{2}$-sentences and hold in every e.c. LF-group by [14, Theorem 6.1] and [4, Theorem 2]. Moreover, (b) follows from an iterated application of the corresponding statement for finite $H$, which in turn can be expressed as an $\forall_{2}$-sentence that holds in the unique countable e.c. LF-group [14, Theorem 6.1].

Of course, one is tempted to ask in how far the assertion (a) of Theorem 6.1 extends to isomorphisms between infinite f.g. subgroups of an e.c. LcF-group $G$. We will show now that it is hardly possible to make much progress in this direction.

Theorem 6.2. Let $G$ be an e.c. LcF-group.

(a) An isomorphism $\phi: A \rightarrow B$ between f.g. subgroups of $G$ is induced by conjugation in $G$, if and only if there exists a local system $\mathscr{L}$ of f.g. subgroups of $G$ which contain $\langle A, B\rangle$ such that, for every $X \in \mathscr{L}$, the topology on $X$ is given by a residual system $\mathscr{R}_{X}$, such that $\phi$ induces an isomorphism $A N / N \rightarrow B N / N$ for every $N \in \mathscr{R}_{X}$.

(b) An element $g \in G$ of infinite order is conjugate in $G$ to $g^{n}$ ( $n \in \mathbb{Z}$ ), if and only if $0\left(g^{n} N\right)=0(g N)$ for every $N \geqq_{0}\langle g\rangle$. In particular, no element of infinite order in $G$ is conjugate to all of its non-trivial powers.

Proof. (a) follows from Theorem 2.3 and [7, Lemma 1].

(b) If $0\left(g^{n} N\right)=0(g N)$ for every $N \geqq_{0} U=\langle g\rangle$, then (a) implies that $g$ is conjugate to $g^{n}$. Conversely, suppose that $0\left(g^{n} N\right) \neq 0(g N)$ for some $N \geqq_{0} U$. Then $0\left(g^{n} M\right) \neq 0(g M)$ for every $M \unlhd_{0} U$ with $M \leqq N$. But every residual system in $U$ contains some $M \geqq_{0} U$ such that $M \leqq N$, whence (a) implies that $g$ is not conjugate to $g^{n}$.

Let $C=\langle c\rangle$ be the infinite cyclic group with the topology given by the residual system of all $N \leqq C$ of finite index. Then Theorem 6.2(b) shows that, in every e.c. LcF-group $G \geqq C$, the element $c$ is conjugate to $c^{n}(n \in \mathbb{Z})$ if and only if $n= \pm 1$.

\section{REFERENCES}

1. B. Baumslag, On the residual finiteness of generalized free products of nilpotent groups, Trans. Amer. Math. Soc. 106 (1963), 193-209.

2. B. Baumslag and M. Tretkoff, Residually finite HNN-extensions, Comm. Algebra 6 (1978), 179-194.

3. J. Dugundu, Topology (Allyn and Bacon, Boston 1966).

4. M. Gerstenhaber and O. S. Rothaus, The solution of sets of equations in groups, Proc. Nat. Acad. Sci USA 48 (1962), 1531-1533.

5. E. S. Golod, On nil-algebras and finitely approximable p-groups, $A M S$ Translations (2) 48 (1965), 103-106.

6. R. I. Grigorchuk, Construction of p-groups of intermediate growth that have a continuum of quotient groups, Algebra and Logic 23 (1984), 265-273. 
7. P. Hall, Some constructions for locally finite groups, J. London Math. Soc. 34 (1959), 305-319.

8. B. Hartley, Profinite and residually finite groups, Rocky Mountain Math. J. 7 (1977), 193-217.

9. F. Haug, Existenziell abgeschlossene LFC-Gruppen (Dissertation, Tübingen 1987).

10. E. Hewitt and K. A. Ross, Abstract harmonic analysis I (Springer, Berlin, 1963).

11. P. J. Higgins, An introduction to topological groups (LMS Lecture Note Series 15, Cambridge, 1974).

12. G. Higman, Amalgams of p-groups, J. Algebra 1 (1964), 301-305.

13. J. HirsChfeld and W. H. WheEler, Forcing, arithmetic, division rings (Springer Lecture Notes 454, Berlin, 1975).

14. O. H. Kegel and B. A. F. Wehrfritz, Locally finite groups (North-Holland, Amsterdam, 1973).

15. F. LeINEn, Existentially closed groups in locally finite group classes, Comm. Algebra 13 (1985), 1991-2024.

16. F. Leinen, Existentially closed LX-groups, Rend. Sem. Mat. Univ. Padova 75 (1986), 191-226.

17. F. Leinen, Existentially closed locally finite p-groups, J. Algebra 103 (1986), 160-183.

18. F. LeINEN, Uncountable existentially closed groups in locally finite group classes, Glasgow J. Math. 32 (1990), 153-163.

19. F. Leinen and R. E. Phillips, Existentially closed central extensions of locally finite pgroups, Math. Proc. Cambridge Philos. Soc. 100 (1986), 281-301.

20. R. C. Lyndon and P. E. Schupp, Combinatorial group theory (Springer, Berlin, 1977).

21. B. Maler, Existenziell abgeschlossene lokal endliche p-Gruppen, Arch. Math. 37 (1981), $113-128$.

22. B. H. Neumann, On amalgams of periodic groups, Proc. Roy. Soc. London (A) 255 (1960), 477-489.

23. L. Ribes, On amalgamated products of profinite groups, Math. Z. 123 (1971), 357-364.

24. E. I. Zelmanov, Solution of the restricted Burnside problem for groups of odd exponent, Math. USSR Izvestiya 36 (1991), 41-60.

25. E. I. Zelmanov, Solution of the restricted Burnside problem for 2-groups, Mat. Sb., to appear.

Fachbereich 17-MathematiK

JoHANNES GUTENBERG-UNIVERSITÁT

SAARSTR. 21

D-6500 MAINZ

Germany 